\title{
Dinámicas relacionales de las transiciones a la vida adulta. Complementariedad entre redes, apoyos $y$ soportes ${ }^{12}$
}

\author{
Dr. Martin Goyette - Universidad de Quebec ${ }^{3}$, Canadá
}

\begin{abstract}
Resumen
¿Qué recursos pueden atraer hacia los jóvenes personas que compongan su red (estructura, composición, etc.)? ¿Qué apoyos reciben los jóvenes de manera efectiva por parte de su entorno (red primaria y secundaria)? ¿Cuáles son las condiciones necesarias para que un apoyo se convierta en un soporte real en las transiciones hacia la vida adulta? Hemos decidido utilizar varios marcos de análisis complementarios al análisis de las redes sociales, para enfocarnos de manera compleja en las transiciones a la vida adulta. Así como extraemos del análisis que los jóvenes deben estar rodeados de un entorno, también es necesario que éste sea de calidad. Por otro lado, además del apoyo como tal, es necesario que el joven lo acepte para que le pueda favorecer en las transiciones.
\end{abstract}

Palabras clave: Redes sociales - transiciones.

\begin{abstract}
What are the resources that can emerge from the actors evolving within the youth's social networks? What are the characteristics of the supports actually directed towards the youth? In which conditions can a support really help the transition from adolescence to adulthood? We have chosen to use complementary analysis frameworks in order to apprehend youth's transitions from a more complex point of view. We can conclude from our analysis that if youth need to be surrounded, the good quality of their social network is essential. Clearly, it goes without saying that youth have to show some opening towards their supports in order for them to have a positive effect on their transition process
\end{abstract}

Key words: social Networks - transitions.

\footnotetext{
1 El autor agradece el financiamiento de los trabajos que conforman este artículo al Fondo Quebequense de Investigación Sobre la Sociedad y la Cultura (concurso Beca de Excelencia para Doctorado), al Instituto Universitario del Centro de Juventud de Quebec (beca de apoyo para la realización de una tesis y beca de apoyo para unas prácticas internacionales), a la Estrategia Nacional para la Prevención del Crimen del Gobierno de Canadá, en colaboración con el Ministerio de Seguridad Pública de Quebec, a la Asociación de Centros de Juventud de Quebec, y al Instituto de Servicios y Políticas de Salud de los Institutos de Investigación en Salud de Canadá (concurso beca de investigación postdoctoral).

2 Traducción realizada por Sandra Corcuera Fortuny

${ }^{3}$ Escuela Nacional de Administración Pública [martin.goyette@enap.ca].
} 
La modificación de las condiciones de entrada a la vida adulta, tras varios decenios observándola, está asociada a la prolongación general de la juventud y a una desincronización de los umbrales de las transiciones a la vida adulta (Bidart, 2006). La inserción de los jóvenes ya no se realiza según un modelo social casi unificado, sino más bien individualizando los recorridos, conformados por pasos hacia adelante y hacia atrás en las diversas transiciones (Gauthier, 2000). Dicha prolongación conduce a esta juventud a ser menos independiente o autosuficiente que la juventud de las generaciones anteriores, ya que vive más tiempo con los padres, que contribuyen a su subsistencia más tiempo, especialmente cuando los jóvenes están estudiando (Maunaye et Molgat, 2003). Por otro lado, este contexto de mutaciones sociales de los últimos decenios induce una dinámica donde la construcción de la relación con uno mismo y con los demás está confusa, ya que las referencias, sin ser inexistentes, están en constante movimiento (Gauthier, 2000). Esta situación es todavía más difícil para los jóvenes que han tenido que abandonar los estudios, como son los numerosos jóvenes en dificultad colocados en acogida por los servicios sociales, ya que son confrontados con modelos sociales que a menudo están fuera de su alcance por estar "reservados" a la escolarización avanzada (Goyette, 2006).

Es por esto que la inserción de los jóvenes en general se ha complicado en varios aspectos; esta dinámica o estos imperativos asociados a la transición a la vida adulta constituyen un desafío mayor para los jóvenes que han tenido dificultades, en especial para los que entran en la vida adulta después de una estancia más o menos prolongada en un entorno de acogida (Goyette, 2007).

La inserción social y profesional de los jóvenes que abandonan un medio sustituto parece más difícil a razón de sus dificultades psicosociales y de salud, su falta de preparación para la vida autónoma, y la ausencia de apoyo a su salida del medio sustituto (Goyette et Turcotte, 2004). Yendo más allá de una visión fatalista sobre la inserción de los jóvenes en dificultad, este texto se concentra en el proceso de la inserción vivido por treinta y un jóvenes que abandonaban el entorno de acogida, y se interesa en el papel de las relaciones sociales de manera retrospectiva.

La inserción es por igual un asunto de significaciones y de reflexividad, es importante no simplemente entretenerse en las dimensiones objetivas del análisis, sino tener en cuenta al mismo tiempo la visión que revela el análisis de la composición de las redes, y la que se apoya en una sociología relacional, subrayando asimismo la relación del joven con los demás. 
Primeramente, hemos querido comprender qué recursos pueden atraer a las personas que componen la red de los jóvenes (estructura, composición, etc.), después, qué apoyos efectivos reciben los jóvenes por parte de su entorno (red primaria y secundaria), y finalmente, cuáles son las condiciones necesarias para que un apoyo se convierta en un soporte real para las transiciones hacia la vida adulta. Decidimos utilizar, en complemento con el análisis de las redes sociales (Bidart et Degene, 2005; Charbonneau, 2003), otros dos marcos de análisis para considerar de manera completa las transiciones a la vida adulta (Goyette, 2006), es decir, la inserción a través de los ciclos de vida (Bidart, 2006) y las diferentes transiciones (Galland, 1991; Coles, 1995), y el marco del actor y su margen de maniobra (Martuccelli, 2002) y de la construcción de la identidad (Martuccelli, 2002; Bajoit et al., 2000). En definitiva, se trata pues de reubicar al individuo en sus relaciones comprendiendo el sentido que éstas tienen para él en la continuidad de una sociología relacional y de soporte (Martuccelli, 2002).

\section{Redes sociales, transiciones a la vida adulta y construcción de identidad}

El hecho de comprender los soportes del individuo es particularmente significativo en el caso de los jóvenes. El paso de la adolescencia a la vida adulta corresponde así a "mutaciones biográficas a menudo combinadas" que no se repiten más tarde en la vida (Bidart, 2006). Además, para algunos jóvenes que se encuentran en un medio sustituto, abandonar definitivamente el centro de juventud ${ }^{4}$ también significa abandonar la única familia y hogar que han tenido (Goyette, 2003). La juventud es pues un momento particularmente crítico de la construcción de la identidad, ya que se encuentra en medio de las negociaciones entre las herencias familiares, el acervo adquirido en la escuela, los aprendizajes de la infancia (Bidart, 1999) y las nuevas vías que permiten la realización de los proyectos personales. En esta perspectiva, las diferentes relaciones sociales de un individuo pueden contribuir a "orientarlo", a construirlo. De hecho, "las personas que lo rodean y la configuración relacional que éstas componen forman un sistema de referencia, un "universo de posibles" donde el joven se va a situar (Bidart, 1999:7). Es más, toda persona que el individuo conoce le abre pequeños mundos (Bidart et Le Gall, 1996) dándole acceso a información,

\footnotetext{
4 Los Centros de Juventud son establecimientos que ofrecen servicios especializados a la juventud en un mismo territorio. Se crearon gracias a una reagrupación de servicios a la infancia (los centros de protección de la infancia y de la juventud surgieron varios años antes que los centros de servicios sociales) y servicios de readaptación para los jóvenes en dificultad (centros de readaptación). Se trata pues de la creación bajo una misma organización de un contínuo en protección de la juventud que se encuentra en delicuencia de menores y readaptación psicosocial. Los centros de juventud tienen por mandato prioritario la aplicación de la Ley de Protección de la Juventud, la Ley sobre el Sistema de Justicia Penal para adolescentes y la Ley de los Servicios de Salud y Servicios Sociales. Los dieciséis (16) centros de juventud de Quebec dan servicio a unos 100.000 niños, jóvenes y familiares en dificultad todos los años.
} 
conocimientos y experiencias, y así durante toda su vida. ${ }^{5}$ Las redes sociales nos permiten pues leer la amplitud de círculos sociales que pueden influir en la inserción social de un joven, en la relación entre individuo y sociedad (Degene et Forsé, 1994). Estas relaciones sociales traen apertura al mundo. "Uno no se hace adulto solo, sino en interacción con este entorno y bajo la mirada de ciertos "otros significativos": sin duda los padres, pero no nada más ellos. Otros ejemplos y modelos están presentes en el entorno y "encarnan" diversas elecciones de vida, también difunden opiniones y consejos susceptibles de pesar sobre las decisiones" (Bidart, 2008, p. 560). Son ejemplos importantes el sentido que le dan los actores a su construcción de vínculos y cómo abren y cierran ciertos accesos a mundos sociales y a recursos a los que están ligados. La utilización de recursos está relacionada pues con las representaciones de los actores.

Además de un marco conceptual que nos conduce a las redes, la segunda perspectiva teórica en la que nos inspiramos lleva a la comprensión analítica de la inserción de los jóvenes a través del ciclo de vida de los individuos, las transiciones se convierten en el elemento central, como en los análisis de Galland (1991) y Coles (1995). Interesándose en las transiciones a la vida adulta de los jóvenes, el análisis se ancla en una lectura sobre el éxito y la reversibilidad de las situaciones haciendo resurgir la calidad de actor de las personas socialmente vulnerables, más que reforzar las imágenes victimizantes 0 incapacitantes generalmente utilizadas para describir su situación. Visto desde esta óptica, la juventud no es únicamente una etapa de "falta de estado adulto", los jóvenes son actores, sujetos que se construyen. Es por esto que, a la manera de Bidart, el término "transición" será el preferido ante las nociones de umbrales y pasajes, demasiado categóricos y "adultocéntricos" (Bidart, 2006). Para Coles (1995), hay tres transiciones que marcan el paso de la vida de adolescente a la de adulto autónomo: la transición de la escuela al trabajo, de la familia de origen a una nueva familia, y de la casa de los padres a un alojamiento independiente. Coles trata de mostrar que estas transiciones son importantes y actúan de manera interdependiente. Ahora bien, a partir de estas transiciones, nos será posible considerar cómo las relaciones sociales las facilitan, incluso si estas transiciones se operaron de forma diferente para los jóvenes que abandonan un medio sustituto que para la juventud en general, especialmente las de la ruptura con el medio familiar (Goyette, 2006).

\footnotetext{
5 Como dice Lahire (1998: 233) los actores atravesaron en el pasado y atraviesan permanentemente múltiples contextos sociales; son fruto (y portadores) de todas las experiencias (no siempre compatibles, no siempre acumulables, y en ocasiones altamente contradictorias) que vivieron en múltiples contextos.
} 
En este marco, este artículo apunta a comprender cómo las redes sociales contribuyen a la inserción social y profesional de los jóvenes en dificultad a partir de las transiciones a la vida adulta. Esta comprensión va más allá del estricto nivel individual de resiliencia, con el fin de comprender las condiciones sociales de ésta última. La estructura y la composición de las redes son importantes en la comprensión del papel de las relaciones sociales en las transiciones a la vida adulta, ya que éstas delimitan los posibles apoyos, es decir, el capital social reticular (Goyette, 2006); por tanto, emitimos la hipótesis de que para que tengan un efecto favorable en las transiciones a la vida adulta, el joven debe concebir sus relaciones sociales como un apoyo, es decir, tener una relación con los demás que le proporcione el apoyo activo en el proceso de inserción. En definitiva, tratamos de reubicar al individuo en sus relaciones comprendiendo el sentido que tienen para él en la continuidad de una sociología relacional y de apoyo (Martuccelli, 2002). La cuestión relacional se convierte así en la manera contemporánea y moderna de hacerse un individuo, y los apoyos son una condición necesaria para que un individuo se inserte socialmente (Martuccelli, 2002, De Singly, 2000). Nuestra problematización teórica responde entonces al marco moderno de la construcción del individuo, que concibe esta construcción como un proceso "por el cual el individuo, por medio de la gestión relacional de sí mismo, (re)construye sin cesar su identidad personal, con vistas a participar en la vida social" (Bajoit et al., 2000:19).

En la comprensión de las dinámicas relacionales, hacemos una distinción entre red social, apoyo y soporte que seguiremos a lo largo de todo nuestro análisis. Analizaremos los recursos potenciales o latentes disponibles en la red de los jóvenes. La red está constituida de personas que el joven nos indica que están en su entorno a partir de las respuestas a los generadores de nombres que se le han presentado (Bidart et Charbonneau, 2007). Se trata de una lectura en términos de capital social reticular, que sigue la línea de los análisis de redes sociales.

Un recurso de una red se vuelve un apoyo cuando se activa, cuando pierde su estatus de recurso estático o latente. Es por ello que también hay que considerar el análisis relacional. Un miembro de la red se convierte en un apoyo en cuanto hay activación o movilización de sus recursos para el joven. Se trata de un nivel de análisis muy diferente: si un individuo no tiene a nadie en su red, es de hecho difícil creer que podrá movilizar sus recursos. Por otro lado, tener una red amplia no es suficiente para que exista una movilización del recurso y que se le proporcione apoyo. $Y$ un apoyo expresado no tiene los mismos efectos según la naturaleza de la relación entre el joven y los actores que le apoyan. Por ello, queremos comprender cuáles son las condiciones que permiten que los apoyos se conviertan en 
soportes significantes en las diferentes relaciones del joven con su red en las transiciones a la vida adulta.

\section{El procedimiento metodológico}

La estrategia metodológica, anclada en un marco comprensivo, es doble en la medida donde liga un análisis de la composición de las redes con un análisis de las significaciones de las relaciones sociales para el joven. En este contexto, se trata tanto de recoger datos objetivos constituidos por la descripción de las relaciones sociales del joven, como de datos subjetivos que nos lleven a las experiencias y al sentido que el joven les atribuye.

\section{La población a estudio}

Los datos utilizados en este estudio se recogieron en el marco de la evaluación de la implantación y los efectos de un proyecto piloto de intervención intensiva enfocada a facilitar el paso a la vida autónoma de jóvenes a cargo de centros de juventud en Quebec (Goyette et al. 2007). El Proyecto Calificación de Jóvenes (PQJ) ${ }^{*}$, implantado en 2001 por la Asociación de Centros de Juventud de Quebec, implicó a ocho tutores, provenientes de cuatro regiones distintas, que aseguraron cada uno el seguimiento de 10 jóvenes durante un periodo de tres años, o sea, de los 16 a los 19 años, cubriendo así el primer año en el que dejaron de estar a cargo de los centros de juventud. Los jóvenes participantes fueron seleccionados entre los perfiles más sombríos de los centros de juventud. Estos jóvenes, en el momento de su entrada al programa, no pudieron o no tuvieron la posibilidad de regresar a su medio de origen cuando finalizó la custodia del centro de juventud al cumplir la mayoría de edad. Todos ellos conocieron en el transcurso de sus vidas una historia importante de colocación en medios sustitutos (al menos dos años). El proyecto se dirige a 3 objetivos específicos: 1) preparar y dirigir el paso a la vida autónoma; 2) integrar a los jóvenes en el mercado laboral o en una formación ocupacional; 3) desarrollar redes de apoyo y ayuda entorno a los jóvenes.

El presente artículo se basa en 31 jóvenes de los 80 que participaron en el proyecto piloto, 14 chicas y 17 chicos. En el momento de la entrevista, los jóvenes tienen entre 18 y 20 años: el 68\% tienen 18 años y el 29\%, 19 años. Todos habían alcanzado la mayoría de edad legal y la custodia de los centros de juventud ya había llegado a su fin. Estaban pues en plena transición hacia la vida adulta, lo que a menudo implicaba requerir autonomía y satisfacer sus necesidades.

* (N. del T.) En francés, «Projet qualification des jeunes » cuyas siglas son PQJ. 


\section{Las fuentes de datos}

Todos los datos sobre los jóvenes fueron recopilados a lo largo de un encuentro en el que se abordaron sucesivamente estos temas: calendario biográfico, entrevista sobre historia de vida, y cuestionario de red.

El calendario biográfico es una herramienta que permite recolectar información desde el nacimiento del joven, sobre la trayectoria familiar, la trayectoria residencial (lugar de residencia, tipo de residencia, miembros del personal doméstico, razón del cambio de lugar de residencia), el historial de colocación en acogida, la trayectoria escolar (naturaleza de la formación, duración de la formación, etc.) y la trayectoria profesional (medios por los que se encontró el empleo, ocupación, horario, horas trabajadas por semana, duración y razón de la marcha) (Charbonneau, 2003, Goyette et al. 2006a, Goyette, 2006).

Las entrevistas del tipo historia de vida permiten que el joven se tome un tiempo para verlo con perspectiva y que él mismo se lo cuente. El procedimiento en estas entrevistas se enfoca a documentar la evolución de la situación del joven a través de diferentes áreas de su vida (inserción en la escuela, trabajo y remuneración, situación financiera, inserción residencial, tiempo libre, inserción en el espacio local, relaciones con los padres, vida amorosa, habilidades y relaciones sociales), y las relaciones sociales que mantiene así como el sentido que les atribuye en el contexto de las transiciones que vive o que vivió.

El cuestionario sobre las redes sociales permite la reconstrucción de las redes sociales y redes de apoyo a partir de generadores de nombres (Bidart et Charbonneau, 2007). Este cuestionario se inspira en el procedimiento original de Bidart (2002), quien se interesa en la construcción de la inserción socioprofesional y en la evolución de las redes sociales de jóvenes franceses. La reconstitución de las redes sociales y de las redes de apoyo se sostiene sobre una estrategia metodológica organizada a partir de la utilización de diferentes tipos de generadores de nombres, que permiten reconstruir el conjunto de personas (los alters) con quien un "ego" está en contacto en diversos contextos de vida. Así, nuestro cuestionario distingue la red de personas significativas (las personas próximas e íntimas, que son fuente potencial de apoyo psicológico) del conjunto de otras relaciones que han desarrollado en diversos contextos presentes y pasados de su vida (Charbonneau et Turcotte, 2005 :11-14; Francke, 2005). Todas las personas cuyos nombres se recogen son objeto de un cuestionamiento complementario destinado a conocer sus características personales y la historia de su vínculo con el ego (edad, sexo, ocupación, situación familiar, 
estatus de empleo, nivel de estudios, lugar de residencia, estatus en relación al ego ${ }^{6}$, antigüedad de la relación, contexto del encuentro, distancia residencial actual, frecuencia de contactos, actividades realizadas juntos actualmente, actividades ya realizadas juntos, etc.).

Este acercamiento nos conducirá a un análisis mixto de los datos que se dedicará por igual tanto al objetivo como al subobjetivo (Huberman et Miles, 1991, Laperrière, 1997). El tratamiento de datos está constituido en un primer tiempo por el crecimiento por parte de cada joven, y en un segundo tiempo, por un tratamiento de sus relaciones y configuraciones sociales. De esta manera, las entrevistas fueron consideradas como unidades de análisis equivalentes.

\section{Los recursos reticulares de los jóvenes}

En la perspectiva en la que comprendemos las redes sociales como reservas potenciales de recursos relacionales, sacaremos a colación ciertos resultados del retrato descriptivo de las redes relacionales de los jóvenes entrevistados. De entrada, hay que mencionar que dichos jóvenes, contrariamente a la imagen mediática y popular, no están aislados, es decir, sin entorno (media de 14'9 alters por ego, con una mediana de 14 y desviación estándar de 5'12, sin diferencia significativa entre sexos). Todos dicen tener personas que consideran importantes en su vida y amigos. De media y sin distinción entre sexos, las redes están compuestas en la mayoría por amigos (56\%) y por miembros de la familia (cercanos o lejanos) (21\%). Los otros alters presentes en las redes son trabajadores sociales (11\%) y conocidos $(9 \%)$, incluyendo vecinos, colegas, jefes, etc. Los cónyuges y ex parejas representan el $4 \%$ de sus redes sociales.

Aunque no estén completamente aislados, estos jóvenes están cuantitativamente menos rodeados que los jóvenes de la población general que nunca han estado en un medio de acogida. Un estudio dirigido por Sylvain Bourdon y Johanne Charbonneau sobre las redes sociales de 96 jóvenes colegiales de la misma edad, aporta que la entendida media de su red social es de 30 alters, dos veces mayor que la de los jóvenes que han vivido dificultades. Los colegiales no solamente tienen más amigos que los jóvenes del PQJ, sino que sus relaciones de amistad son también más antiguas y más homofílicas. Los estudiantes de CÉGEP* tienen más ocasión de escoger amigos parecidos a ellos, especialmente porque los encuentran en el contexto escolar (Poirier et Lavoie, 2006), mientras que los jóvenes del

\footnotetext{
${ }^{6}$ Con el contexto del encuentro, el estatus del alter en relación al ego viene a distinguir la red primaria de la red secundaria.

* (N. del T.) CÉGEP : Collège d'enseignemet générale et professionnelle (Colegio de enseñanza general y profesional). Son escuelas exclusivas del sistema educativo en Quebec a las que se accede después de la secundaria, bien para obtener diplomas vocacionales, o bien para preparar el acceso a la universidad.
} 
PQJ los encuentran más por medio de otras personas. Sus relaciones íntimas son más recientes que las de los jóvenes colegiales. Por otro lado, el hecho de tener varios amigos íntimos entre sus relaciones recientes, "demuestra una sociabilidad problemática. Estas constataciones tienden a confirmar la hipótesis de una sociabilidad particular de los jóvenes que fueron colocados en medios de acogida, marcada [por] (...) una tendencia a considerar las relaciones todavía embrionarias como importantes, en particular en el caso de las chicas." (Poirer et Lavoie, 2006). De ahí la casi ausencia de conocidos en la red de los jóvenes del PQJ (Goyette et al. 2007).

En ciertas variables, nuestros análisis confirman la tendencia a la homofilia en las redes de los jóvenes estudiados (por género para los chicos, por ocupación, para los que están inactivos y estudiando). Sin embargo, es necesario aportar matices en cuanto a la relación entre la edad y el sexo. Los datos indican que las chicas frecuentan alters masculinos mayores que ellas, mientras que los chicos se relacionan con alters femeninos más jóvenes que ellos. La homofilia por edad es pues más fuerte con los alters del mismo sexo. Las chicas frecuentarían pues a otras chicas de su edad y a chicos mayores que ellas, mientras que por el contrario, los chicos se relacionan con chicos de la misma edad y con chicas más jóvenes.

Esta tendencia homofílica es por otro lado menos importante que la observada en otros estudios sobre redes sociales. Una tendencia a la homofilia de las redes en cuanto al género, al origen social, a la edad combinada con la etapa del ciclo de vida, se ha mostrado en diversas investigaciones sobre las redes sociales (Grossetti, 2002; Forsé 1999). Las redes sociales de los jóvenes participantes en el PQJ y las de los jóvenes colegiales han sido comparadas y los resultados revelan que los colegiales tienen redes mucho más homofílicas (en términos de edad y sexo) que los del PQJ (Poirier, Lavoie, 2006). Sin embargo, estos datos permiten mostrar un perfil de sociabilidad diferenciado según el sexo de los jóvenes. En un primer momento, los chicos parecen relacionarse más con alters de su mismo sexo. En un segundo momento, las chicas se unen más a una forma de sociabilidad en la que los chicos ocupan un lugar más importante en su red.

Por otro lado, el patrón tipo de las redes de los chicos estudiados sería más propicio a la inserción y al apoyo. Ellos pueden contar con ciertos recursos de su red. Los jóvenes varones tienen ciertamente un número importante de amigos de su mismo sexo, sus madres están más presentes y los miembros de sus familias citados pueden potencialmente procurarles apoyo. Al final, la red de los chicos se caracteriza por la antigüedad de las relaciones: tienen más relaciones antiguas y más vínculos familiares. Esta antigüedad constituye, según Lavenu (2001), un marcador potente de la activación de los apoyos: no 
colocamos en nuestra red a personas para que nos apoyen, la elección está más ligada a una sociabilidad de ocio. Así, como los análisis de Lévesque (2000) mostraron, podemos creer que las redes más antiguas tienen oportunidades mucho más grandes de ofrecer apoyos instrumentales (alojamiento, empleo) que las que son más recientes. Asimismo, también hay que subrayar que los padres pueden constituir una fuente potencial muy importante de apoyo expresivo e instrumental (Maunaye, 2004; Maunaye et Molgat, 2003). Ahora bien, en el caso en el que los padres no son mencionados por los jóvenes como parte de su red, podemos creer que su apoyo está ausente o es poco significativo. En este contexto, los chicos parecen estar más favorecidos que las chicas, en la medida en la que la madre, que puede desempeñar un papel central, está más presente.

Por su parte, el patrón tipo de las redes de las chicas encierra menos recursos de apoyo. Los análisis realizados a este respecto mostraron que cuantos más chicos se encuentran en la red de las chicas, más se bloquea la transición a la vida adulta de éstas (Goyette et al., 2006b). Además, la mitad (50\%) de las chicas no tienen a sus padres en su red, existe una presencia incrementada de chicos y mantienen en general relaciones más recientes. Asimismo, estas chicas encuentran gente con mayor frecuencia por medio de una tercera persona. Ellas se implican más "en el sentido amoroso" y representan el $89 \%$ de los jóvenes estudiados que viven con su cónyuge. Las chicas suelen encontrar personas nuevas por medio de su cónyuge, que ocupa un lugar central en su red.

Así pues, la red de estas chicas parece tener menos recursos reticulares diversificados 0 susceptibles de actualizarse como soporte en las transiciones. Además, ellas se muestran más cautivas de su red, dominada por una sola persona. Esta falta de diversificación tiene un impacto negativo sobre el potencial de la red social en cuanto a proporcionar recursos múltiples (Grossetti, 2005, 2002; Bidart, 1999).

Además, las redes más heterofílicas corresponden más a chicas que parecen más aisladas socialmente y cuya red está centrada en el cónyuge. Las redes más homofílicas están asociadas sobre todo a chicos insertados en redes de amigos del mismo sexo y más orientados a la diversión (Goyette et al., 2007; Poirier et Lavoie, 2006).

Si la red es homogénea y muy densa, el individuo será fuertemente insertado, pero en este único medio social (Granovetter, 1973), ya que "si se sale de este medio, se arriesga a quedarse sin recursos. Si por el contrario la red es heterogénea y dispersa, y sus miembros se conocen poco entre ellos, el individuo estará menos integrado en un medio, pero también menos dependiente, podrá desplazarse con mayor facilidad, adaptarse a situaciones diversificadas, verse desempeñando diferentes facetas de su identidad" (Bidart, 1999: 9). 
Así pues, como recuerda Lecoutre (2003 : 62) es posible "obtener beneficios de estructuras sociales que tienen formas opuestas", pero las redes cerradas permiten más apoyo social expresivo, mientras que las redes abiertas y diversificadas permiten más una acción instrumental : obtener un empleo, encontrar un alojamiento, etc. (Degene et Forsé, 1994 : 62-65). Así, las redes sociales de las chicas estudiadas son herméticas por estar centradas en el cónyuge, mientras que las redes de los chicos son herméticas a razón de estar centradas o bien en la familia o bien sobre el grupo de amigos, donde se revela una fuerte homofilia.

Por otro lado, el tema es igualmente importante, ya que para la mayoría de los jóvenes esta sociabilidad decaerá desde el momento en que las transiciones a la vida adulta progresen (Grossetti, 2005). Cuanto mayores se hagan estos jóvenes, más posibilidades hay de que los "pequeños mundos" se restrinjan. No se tratará entonces de aislamiento, sino de confinamiento a un grupo social que inscribe al joven en una sociabilidad en función de un medio social, de ahí la importancia de comprender la evolución de esta dinámica de la sociabilidad. Las redes sociales de los jóvenes en dificultad aparecen ya tan centradas en un medio social en particular o en una persona en particular, que la disminución de la diversidad podría en su caso señalar, si no aislamiento, encierro.

El papel que pueden desempeñar los amigos en la red de los jóvenes en general, que constituyen el $56 \%$ de las redes, parece poco definido, tanto para las chicas como para los chicos. Los datos que nos llevan al análisis de las actividades compartidas por los jóvenes y los miembros considerados como importantes en su red, muestran que la sociabilidad permite poco inscribirse en los espacios institucionales portadores de nuevos "pequeños mundos". De hecho, esta sociabilidad en las relaciones con los amigos es poco susceptible de sostener la construcción de la identidad y las transiciones a la vida adulta, bajo el punto de vista del capital relacional. Bidart (2008), que trata la influencias de las redes en las transiciones a la vida adulta, señala que los padres y los cónyuges son las personas más influyentes en las redes sociales de los jóvenes. Si bien se solicita a menudo a los amigos, la influencia real proviene más de los padres y cónyuges, que "se confirman más presentes en la influencia «percibida», mientras que los amigos dominan sobre todo en la influencia « demandada». Así pues, los jóvenes piensan sobre todo en sus relaciones de amistad cuando imaginan un escenario de petición de consejos, sin embargo sus verdaderos apoyos en una situación real hacen aumentar la importancia de los padres y del alter-amor." (Bidart, 2008, p. 564). 
Del mismo modo, la sociabilidad de los jóvenes estudiados parece poco propicia para la construcción de proyectos a partir de actividades extra profesionales, ni siquiera parece suscitar esta capacidad reflexiva sobre su recorrido, especialmente porque los alters con quien comparten esta sociabilidad, en general pertenecen a su mismo ambiente social inmediato. Dicho de otra manera, esta sociabilidad está mucho más inscrita en una forma relacional ligada a la adolescencia. Los amigos considerados importantes son los alters que comparten la mayoría de actividades con ellos, una media de seis actividades diferentes. Las más compartidas con los amigos son: hablar, debatir, y comer juntos (63\%), fiestas en casa de alguien (61\%), bares y discotecas (50\%), ver la televisión (50\%), consumir drogas $(47 \%)$, videojuegos $(42 \%)$, salir a pasear, e ir de tiendas $(42 \%)$, cine y conciertos $(36 \%)$, cafeterías y restaurantes $(31 \%)$, encontrarse en lugares públicos (monumentos, parques, metro) (24\%), y acondicionamiento físico y deporte (24\%).

Así, sin por tanto mencionar la ausencia del papel que desempeñan las transiciones que permiten el paso a la vida adulta, parece que esta sociabilidad no sea estructurante, los "pequeños mundos" a los que da acceso no están asociados por ejemplo a competencias transferibles por un proceso de "socialización transicional" tal como teorizó Roulleau-Berger (1997). Por otro lado, hay experiencias de sociabilidad de los jóvenes, por ejemplo a través de un proyecto o de una actividad cultural, que les permiten inscribirse en procesos de inserción. Esta sociabilidad puede convertirse en un preámbulo que a veces apoya las transiciones que permiten el paso a la vida adulta (Le Gall, 1999). De la misma forma, en la literatura sobre la juventud, los ejemplos del Hip Hop y las culturas alternativas se suelen analizar como emblemáticas de esta adquisición de competencias, que más tarde son transferibles en una forma de inserción al trabajo (Vulbeau, 2003); no obstante, éste no es el caso de los jóvenes que hemos estudiado. Finalmente, la sociabilidad de estos jóvenes no parece tampoco permitir una mejor influencia de los otros jóvenes sobre sus vidas (conocimiento de la actualidad, actitud menos pasiva, investigación de información y trámites administrativos autónomos, en ocasiones incluso por teléfono, capacidad de anticipación, etc.) tal y como señaló Le Gall (1999) de los jóvenes que él estudió.

En resumen, si estos jóvenes no están aislados stricto sensu, parece que esta forma particular de sociabilidad es importante para los jóvenes en dificultad, ya que no permite precisar un proceso de identidad. Las prácticas de sociabilidad observadas revelan así para nosotros un proceso exploratorio y difuso, no inscrito en las transiciones a la vida adulta. Por otro lado, si el análisis de la composición de las redes hasta aquí orienta hasta cierto punto hacia los posibles accesos a recursos reticulares, no podemos dejar de fijarnos en las situaciones concretas en las que estos posibles entran en escena. Así, las siguientes partes 
analizarán en una perspectiva complementaria cuáles son los soportes que realmente se aportan a los jóvenes y cómo contribuyen a las transiciones, verdaderamente o no, permitiendo las transiciones a la vida adulta. Será pues necesario comprender de qué manera se activa este capital relacional para favorecer la inserción del joven.

\section{Los actores y la naturaleza de los apoyos en las transiciones}

Cuestionarse sobre la naturaleza, los contextos y los actores que muestran apoyos en las tres transiciones hacia la vida adulta de los jóvenes en el estudio, permite comprender mejor el papel de las relaciones sociales en dichas transiciones. Para ello, hemos reinterpretado el relato subjetivo de la entrevista del joven sobre su experiencia social de apoyo para clasificarlo en categorías de experiencia más formalizadas. Estos datos no se nos proporcionaron como tales a priori, sino que elaboramos nuestro análisis a partir de un trabajo de formalización a posteriori a partir del informe de los jóvenes sobre su experiencia social de apoyo. El objetivo de este estudio no es pretender una relación exhaustiva de apoyos recibidos por los jóvenes participantes, sino más bien llevar la atención a los que se han impuesto como importantes para ellos y adquirieron sentido en su experiencia social de apoyo, y que a su vez han elegido compartir con nosotros. Hemos querido que sea comprensible para el lector la representación de la experiencia social de apoyo de estos jóvenes, a través de la presentación jerarquizada de las categorías de actores y de apoyos importantes. De esta manera, tratamos de hacer comprensible la complejidad de los procesos sociales en el estudio.

Sin lugar a dudas, el tutor del proyecto piloto en el que estos jóvenes en dificultad están inscritos, es de lejos el actor que más apoya de la red. Sin contar a este tutor, la familia inmediata es la categoría de actores que más apoyo da a los jóvenes, seguida de los servicios sociales de readaptación, y después, del resto de las instituciones. Los amigos y la familia de los amigos, el resto de la familia, el cónyuge y la familia del cónyuge, se vuelven figuras de apoyo en proporciones equivalentes. Los apoyos recibidos restantes provienen de los demás actores presentes en la vida del joven (conocidos, vecinos, compañeros de trabajo, jefe, grupos comunitarios y otros recursos alternativos del medio), que son poco numerosos ${ }^{7}$.

\footnotetext{
${ }^{7}$ Representan el 9\% del total de las redes de los jóvenes; 17 jóvenes de 31 cuentan al menos con una persona en su red que se categoriza entre estos « otros actores » y solamente 5 jóvenes cuentan con más de dos.
} 
Ciertos actores están más presentes en ciertas transiciones, por ejemplo, los padres (cuando están presentes en la red) resultan de mucho apoyo en general en el área del alojamiento ofreciendo una ayuda material, pero están mucho menos presentes en el apoyo a la transición profesional y familiar. Además, el apoyo de un actor se activa en ocasiones según los acontecimientos. También hemos constatado que aunque la mayoría de los actores no apoyan la transición hacia una nueva familia, cuando una joven queda embarazada moviliza los apoyos de su entorno para apoyarla en esta transición, en detrimento, por otro lado, de los apoyos a la transición profesional. La movilización de un apoyo material y en ocasiones emotivo por parte de la madre, es también un buen ejemplo de ello. Si bien las situaciones varían de un joven a otro, el tutor del proyecto piloto siempre es un actor de primer plano que actúa en complementariedad con los otros actores de la red para apoyar al joven.

Sin embargo, no todos los apoyos desempeñan el mismo papel en las transiciones a la vida adulta. Nos hemos interesado pues en las acciones que les permiten convertirse en soportes partiendo de la singularidad de las situaciones, para acabar discerniendo cuáles fueron los efectos de estos apoyos sobre las transiciones a la vida adulta.

\section{Las diferentes acciones del apoyo}

Respecto a las transiciones a la vida adulta, considerar las diferentes acciones del apoyo constituye una ocasión de distinguir los que refuerzan las transiciones de los que las bloquean. De hecho, que exista un apoyo no significa necesariamente que tenga un efecto positivo sobre las transiciones de los jóvenes a la vida adulta. En otros términos, se trata de mostrar en qué situación el apoyo es bello y de veras efectivo. En esta perspectiva, para llegar a calificar estas formas de acción diferentes, hemos elaborado un marco distintivo de estas acciones, considerando que los apoyos pueden ser inhibidores, vectores o puentes. Así, los diversos apoyos están ilustrados por estas tres figuras que asocian a los diferentes actores entorno a los atributos de participación de la acción. Entre las figuras de acción, la del inhibidor está inscrito en una relación con el otro donde la consideración por la transición está ausente o es inadecuada. En cierta forma, es lo que podríamos llamar "malas influencias". Los actores, por su parentesco con el joven, ofrecen modelos o consejos que vienen a bloquear o perjudicar la inserción en una o varias transiciones. La figura del vector es la acción que tiende a apoyar al joven impulsándolo o dirigiéndolo hacia sus procesos cuando se enfrenta a una transición. La acción la realiza el joven, recibiendo siempre el apoyo. Se le muestra el camino a seguir en un proceso de concertación con él a manera de apoyo. Finalmente, la figura del actor puente califica la acción que tiende a tomar en cuenta al joven, más que de encargarse de él; el apoyo entra en una perspectiva de acompañamiento para concretar el proyecto. Un actor puente remite al joven a otros 
actores, lo acompaña hacia otros recursos de manera que favorece sus procesos de inserción.

\section{Apoyos y soportes: la relación del joven con los apoyos}

Para comprender cómo estas acciones de apoyo consiguen convertirse en soporte, todavía hay que precisar cómo recibe el joven estos apoyos que se le ofrecen o cómo se resiste a los obstáculos que le crean estos mismos apoyos. De hecho, cada una de estas figuras de la acción se inscribe en una dinámica relacional particular de la relación del joven con los diferentes actores de su red. Así, un apoyo expresado no tiene las mismas repercusiones según la naturaleza de la relación entre el joven y el actor que le apoya. Queremos analizar cuáles son las condiciones que permiten que los apoyos se conviertan en soportes en las diferentes relaciones del joven con su red para las transiciones a la vida adulta, y así mostraremos la importancia de la complementariedad del trabajo de los actores. El análisis de los diferentes tipos de relaciones que mantienen los jóvenes con sus apoyos respecto a las diferentes transiciones que viven, nos ha permitido esclarecer tres figuras de la relación del joven con su red: en dichas figuras se despliega una dinámica de dependencia, independencia o interdependencia. Cuando el joven encarna una figura de dependencia con su red y sus apoyos, la relación es no recíproca. Si la red le ayuda, el joven no utiliza esta ayuda para moverse hacia la autonomía, sino que sólo acepta la ayuda. A esto le sigue una relación disimétrica, entre alguien que ayuda y alguien que es ayudado, en la cual el joven suele ser infravalorar. Un joven depende de su apoyo cuando la ayuda de este último se vuelve indispensable para asegurar su avance o el mantenerse en una de las transiciones. El joven deja que se ocupen de él y esto le parece necesario y primordial. Siendo así, los apoyos no pueden convertirse en soportes, sino que más bien actúan como muletas.

Una dinámica de independencia contribuye a colocar al joven en un marco en el que éste ignora los apoyos potenciales que tiene, con el fin de organizar él mismo su emancipación en una lógica de autonomía natural. Interiorizando así una figura mítica de la autonomía sin vínculo, el proyecto del joven es convertirse en adulto por sí solo, sin el apoyo de los demás, impermeable a las influencias externas, buenas o malas. La estricta utilización de los recursos sin más inversión relacional por parte del joven, ilustra asimismo esta dinámica de independencia. 
Finalmente, una dinámica de interdependencia aplica la reciprocidad de los intercambios entre jóvenes y sus apoyos. El joven piensa en su proyecto ligado a su red, y no él solo, ya que ha desarrollado por su capacidad reflexiva una preocupación por sí mismo a través de los demás y de los demás a través de sí mismo. En una dinámica de interdependencia, sin perder su propia identidad, los actores estarán en cierta forma contaminados los unos por los otros. Esta dinámica permite tomar en cuenta de manera consciente tanto sus ventajas como sus debilidades, y es donde los apoyos pueden convertirse verdaderamente en soportes.

Al joven tan sólo le basta contar con uno o varios apoyos para que esta acción reconfortante favorezca las transiciones a la vida adulta. Así, un apoyo se convierte en un soporte desde que la relación del joven con el apoyo permite una adecuación. Se trata de alcanzar cierta reciprocidad entre actor-acción de apoyo y el objeto del apoyo. De hecho, hay que fijarse también en que el juego del apoyo depende tanto del actor que apoya como del actor al que va dirigido este apoyo. Este análisis dinámico permite especialmente mostrar que la ayuda en sí misma no aporta nada o poco en la inserción de los jóvenes si no se traduce y no transmite una verdadera dinámica y relación social de integración. Sabiendo que la integración aquí significa realizar o implicarse en un proyecto, es decir, emprender un proceso que tenga sentido tanto para el que da su apoyo como para el que lo recibe, existe una necesidad de inscribir la ayuda en una dinámica de complementariedad y reciprocidad. Por ejemplo, un actor fuertemente inhibidor, es menos dañino en la inserción para el joven que mantiene una dinámica de independencia que para uno que mantiene una de dependencia. En el mismos sentido, un actor vector tiene menos impacto positivo en el joven que se encuentra en una dinámica de independencia e incluso de dependencia, que en una dinámica relacional de interdependencia. Esto subraya la importancia de considerar la influencia de la red, especialmente desde un punto de vista dinámico, pero también hay que tomar en cuenta el margen de maniobra que el joven tiene de aceptar y de negociar los recursos de la red a fin de continuar con sus proyectos. En el mismo sentido, la intensidad afectiva de la relación está ligada a la permeabilidad de las influencias.

Por otro lado, la influencia de un actor y la dinámica relacional mantenida con él pueden variar según las diferentes transiciones. La presencia de un cónyuge puede ser vector para establecer un alojamiento autónomo en una dinámica de interdependencia, desde el momento en el que ambos cónyuges participan activamente y de mutuo acuerdo para mantenerlo. Sin embargo, este mismo cónyuge puede frecuentar amigos que consuman drogas, cuya presencia en la vida de una joven tiene un impacto inhibidor en su transición profesional poniendo en riesgo su perseverancia escolar. 


\section{Dinámicas relacionales e inserción en las transiciones}

Por último, se trata de clasificar el conjunto de estos jóvenes para revelar la acción del apoyo y la relación del joven con este apoyo, con respecto a las diferentes transiciones que viven. Así pues, para cada joven hemos recopilado los diferentes apoyos para ver cuáles eran los predominantes respecto a las diferentes transiciones y los hemos asociado a la relación dominante que el joven mantenía con sus apoyos. Constatamos que la mayoría de los apoyos que reciben los jóvenes se quedan como inhibidores y no permiten entonces apoyar el desarrollo de los proyectos de inserción. Además, cuando la acción de apoyo es más positiva (vector o puente), a penas en raras ocasiones alcanza su pleno potencial de apoyo, ya que los jóvenes se inscriben con mayor frecuencia en dinámicas de dependencia (sobre todo las chicas) o de independencia (más los chicos).

No es muy sorprendente que los jóvenes estudiados se inscriban en una relación de independencia o de dependencia más que en una interdependiente. Estos jóvenes en dificultad parecen no querer confiar en los demás, sino más bien construirse a partir de ellos mismos en una lógica de autonomización, que les será difícil asumir plenamente. De hecho, la reciprocidad que es la base de la interdependencia se apoya en habilidades relacionales que se desarrollan con el tiempo, por ejemplo en el marco de las relaciones familiares o de intercambios en generaciones enteras. Considerando la posición social de estos jóvenes y las bifurcaciones importantes que han conocido y conocen, es sin duda mucho pedir que se inscriban en la interdependencia.

Para favorecer la inserción social y la construcción de un individuo autónomo, es tan necesario que el joven aprenda a relacionarse consigo mismo y con los demás, como con las acciones que le apoyan. Estas condiciones se asocian pues a la vulnerabilización de las transiciones a la vida adulta, o al contrario, a la inscripción en una perspectiva constructiva.

Como los jóvenes no están dotados del mismo capital relacional y de las mismas capacidades para entrar en una relación, hay que señalar que ciertos eventos importantes vividos por varios chicos y chicas parecieron influir en su relación con el ámbito relacional. Sus sentimientos de confianza y seguridad cedieron su lugar al miedo y la ansiedad ante la transición a la vida adulta. Las dificultades emocionales y los déficits en las competencias sociales de estos jóvenes son particularmente importantes cuando se les recoloca después de mucho tiempo y cuando se les ha trasladado de entorno en múltiples ocasiones, sabiendo que estos numerosos traslados tienen impactos diferentes según el momento en el que el joven se encuentra de su trayectoria. En efecto, hay que comprender que las fracturas relacionales sufridas durante el periodo precedente a su custodia por los servicios sociales 
(sobre todo cuando se trata de una relación de violencia física o sexual) contribuyen a veces a inscribir al joven en un sufrimiento relacional que le encierra en una relación consigo mismo y que a su vez orientará su relación con los demás, probablemente en una dinámica de dependencia con su entorno.

Esta dependencia sucede más en las chicas que interaccionan con los diferentes apoyos, viven un proceso de vulnerabilización de las transiciones a la vida adulta, un proceso concebido casi exclusivamente entorno a la transición familiar y en el contexto del encierro relacional explicado anteriormente. Por tanto, ellas no solamente se incluyen en un modo tradicional de paso a la vida adulta centrado en la familia, caracterizado por la división sexual de los papeles sociales donde el hombre es el proveedor, sino que además se encuentran en una dinámica de vulnerabilización donde el aislamiento relativo reenvía en ocasiones a situaciones de violencia sufridas o realizadas.

De la misma forma, los chicos que funcionan en dependencia con los apoyos de su red viven una vulnerabilización de sus transiciones a la vida adulta en la medida en que el joven utiliza esta ayuda a modo de supervivencia y necesidad, y no para incluirse en un movimiento hacia la autonomía, sin considerar los otros apoyos que podrían favorecer una transición. Seguidamente, se da una relación de poder disimétrico entre un ayudante y un ayudado, en la que el joven es infravalorado con frecuencia. Cuando la relación con los demás en una dinámica de dependencia contribuye a incluir el apoyo en una perspectiva vulnerabilizante de las transiciones a la vida adulta, la construcción del individuo autónomo se ve amenazada.

Por el contrario, un joven que se relaciona en independencia con sus apoyos contribuye a construir un proyecto de vida para él y sin los demás. Directamente incluido en una corriente dominante en el imaginario colectivo que ve al individuo moderno autosuficiente, a menudo el proyecto principal de estos jóvenes es el trabajo. Sin embargo, si aprovechan sus apoyos incluyéndose en una transición profesional, no necesariamente aprenden de esta relación con los demás. Este modo relacional de la independencia es incluso a veces fomentado por las estructuras sociales, a fin de reducir la dependencia de los servicios estatales. Bajo esta perspectiva, la concepción habitual de la transición a la vida adulta se basa en una visión normativa del desarrollo de un individuo, en el que la autonomía financiera ocupa un lugar preponderante como resultado a alcanzar (Goyette et Turcotte, 2004). 


\section{¿Independencia o autonomía? Discusión de los resultados}

Si bien las redes sociales se encuentran en medio de las transiciones a la vida adulta y de la construcción de su integración social, el análisis ha mostrado que ciertas condiciones favorecieron la aparición de ciertos tipos de vínculo social. Aquí lo constatamos, la cuestión de las relaciones sociales está mezclada con la de la inserción, porque las relaciones sociales deben pre existir antes que una inserción. Así, para Castel (2003b : 46) "además de las diferencias de capacidades propias de los individuos en el plano psicológico, que podemos hacer la hipótesis de que se reparten de manera aleatoria, [el éxito en un contexto de individualización de trayectorias e incertidumbre] depende fundamentalmente de los recursos objetivos que estos individuos pueden movilizar y de los apoyos con los que pueden contar para asumir las situaciones nuevas". En esta línea, es tan necesario tomar en cuenta los recursos reticulares que posee el joven en su red relacional, como indispensable es considerar la relación del joven con su red.

Por otro lado, en la mayoría de los programas destinados a apoyar a los jóvenes con dificultades a su salida de un medio sustituto, se preconiza esta imagen de un joven autosuficiente. Maluccio et al., (1990), Collins (2001) et Propp et al. (2003) subrayan con pertinencia que existe un sinsentido al hablar de independencia absoluta y completa, situación propia de un mito que por otro lado hace imposible el replanteamiento del problema de la dependencia (Smith, 2001). Castel (2003b : 13) cree muy adecuadamente que una sociedad compuesta de individuos perfectamente independientes no sería una sociedad, sino un "estado sin ley, sin derecho, sin constitución política y sin instituciones sociales". El vínculo social es un componente complejo de la relación humana, el joven en transición siempre está en cierta manera dependiendo de su entorno (Godbout et Charbonneau, 1996) (como el entorno de su ubicación) lo que no significa por tanto la ausencia de autonomía (De Singly, 2000 : 15), sino que se refiere más bien a lo que llamamos aquí interdependencia.

Maluccio et al. (1990) preconizan pasar del registro de independencia al de interdependencia. El concepto de interdependant living está basado en la afirmación que dice que los seres humanos son sociales e interdependientes, es decir, capaces de relacionarse y funcionar con los demás, de utilizar la influencia y los recursos comunitarios. Ser interdependiente significa poder gestionar las tareas de la vida cotidiana y tener una calidad de vida productiva a través de interacciones positivas y apropiadas con individuos, grupos, organizaciones y sistemas sociales. Esto significa reconocer los valores de la mutualidad y la autodeterminación y poder asumir la responsabilidad de las elecciones 
individuales y sus consecuencias (Maluccio et al., 1990). Este concepto permite igualmente restituir cierto poder del actor sobre el joven, para que éste pase de un estatus de objeto de intervención a un estatus de sujeto competente y capaz de utilizar las oportunidades, los márgenes de maniobra y los recursos de su entorno (Collins, 2001; Maluccio et al., 1990). Para Maluccio (1990) se trata de alcanzar la interdependencia que conduce a la autonomía. La inserción real se encuentra bajo esta premisa vinculada con lo relacional, con el vínculo social de calidad.

Desde esta perspectiva, para favorecer la integración plena y entera de los jóvenes en dificultad, es necesario que se encuentren en relación y que esta relación sea de calidad. Y según el modelo de Martuccelli (2002), como ya mencionamos antes, la cuestión relacional en esta perspectiva de interdependencia se convierte en esta forma contemporánea y moderna de hacerse un individuo, siendo los soportes una condición necesaria para que un individuo se integre socialmente. Solamente bajo esta óptica de interdependencia el joven puede construir proyectos de vida fuertes, vinculado a otros, en una perspectiva de reciprocidad.

Así, cuando insistimos en la importancia del desarrollo de una relación con los demás de los jóvenes insertados en una dinámica de interdependencia, nos lleva forzosamente a la necesidad de que el joven esté rodeado, y que en su red haya recursos disponibles que puedan facilitar la inserción, y por último que estos puedan activarse como apoyos. Es más, para apoyar al joven es necesario que la acción que lo sostenga contribuya a que se ponga en marcha. Así, hemos observado cómo un apoyo puede contribuir a bloquear la transición, cuando se comporta como inhibidor. Dentro de este contexto, las condiciones para que se produzca el vínculo social cuando se origina una integración real deben incluir a la vez una relación de interdependencia del joven con su red y una acción que verdaderamente apoye.

\section{Conclusión}

Este artículo se planteó como objetivo general comprender el papel de las relaciones sociales en la transición a la vida adulta de jóvenes que vivieron en acogida. Los procesos de las transiciones a la vida adulta de estos jóvenes están inscritos en una relación consigo mismos y con los demás, lo que atestigua dificultades personales y familiares y una falta de preparación para la vida adulta. La imagen popular de estos jóvenes es una imagen de desafiliación y desarraigo, entre víctima y delincuente. Desde entonces, se plantearon para nosotros los entresijos de las redes sociales. Si estos jóvenes tienen dificultades, cómo va su entorno a sostenerles en el proceso de cambios importantes, cuando además ya sabemos que son cada vez más complejos y largos para todos. Decidimos utilizar varios marcos de 
análisis complementarios al análisis de las redes sociales, para concentrarnos de manera compleja en las transiciones a la vida adulta. Por un lado, una visión cuantitativa de los recursos potenciales, por otro, una visión de los apoyos realmente activos por los actores de la red, y finalmente una lectura de la función de estos apoyos en las transiciones a la vida adulta. Interesarse en los actores y las acciones que favorecen a las transiciones hacia el paso a la vida adulta exigía primeramente considerar cuáles son las redes sociales de estos jóvenes, y sobre todo cómo podían ser una fuente de capital social relacional. Esta visión es esencial, ya que los tipos de recursos que se pueden movilizar dependen de la estructura y la composición de las redes.

Tras analizar de manera integrada las redes, consideramos después cuáles son los apoyos movilizados en estos jóvenes. Entre red y apoyo, existe necesariamente un desfase, ya que los recursos reticulares no siempre se activan para convertirse en apoyos. Así, los miembros de las redes de estos jóvenes no parecían siempre facilitar las transiciones a la vida adulta.

El hecho de insistir en estos dos aspectos de la relación joven-soporte y acción que apoya, recoloca al joven en una posición de actor en la que no se contenta con disponer de los apoyos, sino que negocia unos accesos así como en ocasiones rechaza otros. De esta misma forma, en un contexto de mutaciones sociales y económicas, nuestros análisis demuestran el fracaso relativo del hombre independiente, en la medida en la que él será más o menos permeable de cara a los apoyos de los que es objeto, pero también del que podrá ser el sujeto. Hemos constatado pues que ciertos chicos y algunas chicas se inscriben en esta dinámica; aunque están inscritos con más frecuencia en una transición profesional, se encuentran de todas formas en una situación precaria y no conciben la relación con los demás en una perspectiva de apoyo. Ocurre muy a menudo que su entorno, ya sea de la red primaria o secundaria, incita al menos implícitamente a esta relación de independencia con los demás: el pariente, la sociedad en general, y en ocasiones los programas especializados. Asimismo, para algunas madres jóvenes, desenvolverse por uno mismo es clave. Convertirse en madre sola no construye autonomía, porque autonomía significa interdependencia, más que "autosuficiencia quimérica". Bajo esta perspectiva, es necesario salir de una noción de cantidad, y pensar en los soportes de los jóvenes en transición en términos de calidad. Lo hemos constatado, no basta con querer poner recursos en la red de los jóvenes, hay que incluir recursos de calidad. Además, se requiere que el joven tenga una relación con los demás que permita formar lazos interdependientes. Por ejemplo, hemos mostrado cómo la sociabilidad de los jóvenes, en lugar de constituir un espacio de socialización transicional abriendo el acceso a la adquisición de competencias transferibles eventualmente en una transición profesional, revelaba más una motivación recreativa, una 
sociabilidad adolescente basada en la diversión. En efecto, construir con los jóvenes proyectos en grupo que tienen sentido y les da un lugar, podría permitir a la vez contribuir a romper el universo "cerrado" de los entornos de los jóvenes, haciéndoles descubrir otros "pequeños mundos" y así inscribirlos en un movimiento constructivo hacia las transiciones a la vida adulta. Se trata de constituir las condiciones estructurales y relacionales que crean las oportunidades de inserción, a fin de que seguidamente las condiciones individuales puedan permitir la transición a la vida adulta que da fe de una lógica de integración.

\section{Referencias bibliográficas}

Bajoit G., Digneffe F., Jaspard J.-M. et Nolet De Brauwere Q., 2000.- Jeunesse et société. La socialisation des jeunes dans un monde en mutation, Bruxelles, De Boeck \& Larcier.

Barth R., 1990.- "On their own: The experience of youth after foster care", Child and Adolescent Social Work Journal, 7, pp. 419-440.

Bidart C., 1999.- "Se lier et s'orienter", Agora - débats/jeunesse, 17, pp. 7-18.

Bidart C., 2002.- La construction de l'insertion socio-professionnelle des jeunes à l'épreuve du temps. Une enquête Iongitudinale, Marseille, Laboratoire d'Économie et de Sociologie du Travail.

Bidart C., 2006.- Devenir adulte aujourd'hui : perspectives internationales, INJEP, Collection débats-Jeunesse, L'Harmattan.

Bidart C. et Degenne A., 2005.- "Introduction : The dynamics of personal networks", Social Networks, 27, 4, pp. 283-287.

Bidart C. et Le Gall D., 1996.- "Les jeunes et leurs petits mondes, relations, cercles sociaux, nébuleuses", Mobilités et insertions sociales, spatiales et temporelles, Caen, Cahiers de la MRSH, 5, pp. 57-76.

Castel R., 2003b.- L'insécurité sociale. Qu'est-ce qu'être protégé?, Paris, Seuil.

Chabonneau J., 2004.- Les réseaux sociaux: l'approche relationnelle, Communication présenté au "Projet de recherche sur les politiques. Atelier d'experts sur la mesure du capital social", juin.

Charbonneau J., 2003.- Adolescentes et mères : histoires de maternité précoce et soutien du réseau social, Ste-Foy, Les Presses de l'Université Laval. 
Charbonneau J. et Turcotte M., 2005.- Les réseaux sociaux. Dans Bernard P., Bourdon S., Chabonneau J., Contandriopaulos A. P., Drapeau A., Helly D., Levebvre P. et Paquet G. (Eds.) "Connaître, débattre et décider. La contribution d'une enquête socioéconomique et de santé intégrée et longitudinale (ESSIL)", Montréal, INSPQ.

Coles B., 1995.- Youth and Social Policy: Youth Citizenship and Young Carreers, London, UCL Press.

Collins M. E., 2001.- "Transition to Adulthood for Vulnerable Youth: A Review of Research and Implications for Policy", Social Service Review, 75, 2, pp. 271-291.

De Singly F., 2000.- "Penser autrement la jeunesse", Lien social et politiques - RIAC, 43, pp. 9-21.

Degene A. et Forsé M., 1994.- Les réseaux sociaux, Paris, Armand Colin.

Degenne A., Lebeaux M.-O. et Lemel Y., 2006.- Le capital social dans la vie de tous les jours. Dans Bevort A. et Lallement M. (Eds.) "Le capital social", Paris, Éditions La Découverte.

Forsé M., 1999.- "Âges et sociabilité", Agora - débats/jeunesse, 17, pp. 19-28.

Franke S., 2005.- Measurement of Social Capital Reference. Document for Public Policy Research, Development and Evaluation, Ottawa, PRI Project Social Capital as a Public Tool.Government of Canada.

Galland O., 1991.- Sociologie de la jeunesse. L'entrée dans la vie adulte, Paris, Armand Collin.

Gauthier M., 2000.- "L'âge des jeunes : Un fait social instable", Lien social et politiques RIAC, 43, pp. 23-32.

Godbout J. T. et Charbonneau J., 1996.- La circulation du don dans la parenté: Une roue qui tourne, Montréal, Institut national de la recherche scientifique (INRS-Urbanisation).

Goyette M., 2003.- Portrait des interventions visant la préparation à la vie autonome et l'insertion socioprofessionnelle pour les jeunes des centres jeunesse du Québec, Montréal, Association des centres jeunesse du Québec.

Goyette M., 2006.- Réseaux sociaux, soutiens et dynamiques des supports dans le passage à la vie adulte : le cas de jeunes ayant connu un placement, Ste-Foy, Université Laval. 
Goyette M., 2007.- "Promoting autonomous functioning among youth in care: A program evaluation", New Directions for Youth Development: Theory, Practice and Research, 113, pp. 81-105.

Goyette M., Bellot C. et Panet-Raymond J., 2006b.- Le projet Solidarité Jeunesse. Dynamiques partenariales et insertion des jeunes en difficulté, Québec, Les Presses de I'Université du Québec. Collection Problèmes sociaux et interventions sociales.

Goyette M., Chénier G., Noël V., Poirier C., Royer M.-N. et Lyrette É., 2006a.- Comment faciliter le passage à la vie adulte des jeunes en centre jeunesse. Évaluation de l'intervention réalisée du projet d'intervention intensive en vue de préparer le passage à la vie autonome et d'assurer la qualification des jeunes des centres jeunesse du Québec, Montréal, Rapport de recherche remis à l'Association des centres jeunesse du Québec et au Centre national de prévention du crime.

Goyette M., Royer M.-N., Noël V. et Chénier G., 2007.- Projet d'intervention intensive en vue de préparer le passage à la vie autonome et d'assurer la qualification des jeunes des centres jeunesse du Québec. Rapport final d'évaluation, Montréal, Soumis au centre national de prévention du crime et à l'Association des centres jeunesse du Québec.

Goyette M. et Turcotte D., 2004.- "La transition vers la vie adulte des jeunes qui ont vécu un placement: un défi pour les organismes de protection de la jeunesse", Service social, 51, 1,pp. 30-44.

Granovetter M. S., 1973.- "The Strength of Weak ties", American Journal of Sociology, pp. 78.

Grossetti M., 2002.- Relations sociales, espace et mobilités, Toulouse, Université de Toulouse-le-Mirail.

Grossetti M., 2005.- Réseaux sociaux et hiérarchies sociales : la "ségrégation douce", Aixen-Provence, Communication présentée lors du séminaire Aix, LEST, le 24 mai 2005.

Huberman A. M. et Miles M. B., 1991.- Analyse des données qualitatives - Recueil de nouvelles méthodes, Bruxelles.

Laperrière A., 1997.- La théorisation ancrée (grounded theory): démarche analytique et comparaison avec d'autres approches apparentées. Dans Poupart J., Deslauriers J.-P., Groulx L.-H., Laperrière A., Mayer R. et Pirès A. (Eds.) "La recherche qualitative. Enjeux épistémologiques et méthodologiques", Montréal, Gaëtan Morin.

Lavenu D., 1999.- "Formes de la sociabilité", Agora - débats/jeunesse, 17, pp. 29-46. 
Lavenu D., 2001.- "Activités du temps libre et sociabilité des jeunes à la sortie de l'adolescence", Loisir et société, 24, 2, pp. 403-430.

Le Gall D., 1999.- "Quand la passion déborde le loisir..." Agora - débats/jeunesse, 17, pp. 63-78.

Lecourtre M., 2003.- Capital social, école et entreprises sur le marché du travail, Paris, Université Paris 5.

Lévesque M., 2000.- Le capital social comme forme sociale de capital: reconstruction d'un quasi-concept et application à l'analyse de la sortie de l'aide-sociale, Montréal, Université de Montréal. Thèse présentée à la faculté des études supérieures en vue de l'obtention du grade de Philosophie Docteur en sociologie.

Maluccio A. N., Krieger R. et Pine B. A., 1990.- Adolescents and Their Preparation for Life After Family Care: An Overview. Dans Maluccio A. N., Krieger R. et Pine B. A. (Eds.) "Preparing Adolescents for Life after Foster Care. The Central Role of Foster Parents." Washington, DC, Child Welfare League of America, Inc. Include bibliographical references.

Martuccelli D., 2002.- Grammaire de l'individu, Paris, Éditions Gallimard.

Maunaye E., 2004.- Quitter sa famille d'origine. Dans Pugeault-Cicchelli C., Cicchelli V. et Ragi T. (Eds.) "Ce que nous savons des jeunes", Paris, Presses Universitaires de France.

Maunaye E. et Molgat M., 2003.- Conclusion. Dans Maunaye E. et Molgat M. (Eds.) "Les jeunes adultes et leurs parents. Autonomie, liens familiaux et modes de vie", Sainte-Foy, Les Presses de I'Université Laval et I'IQRC.

Propp J., Ortega D. M. et NewHeart F., 2003.- "Independence or Interdependence: Rethinking the Transition From "Ward of the Court" to Adulthood", Families in Society, 84, 2, pp. 259-266.

Roulleau-Berger L., 1997.- "L'expérience de la précarité juvénile dans les espaces intermédiaires", Formation et emploi, 15.

Singly F., 2000.- "Penser autrement la jeunesse", Lien social et politiques - RIAC, 43, pp. 922.

Smith S. R., 2001.- "Distorted Ideals: The "Problem of Dependency" and the Mythology of Independent Living", Social Theory and Practice, 27, 4, pp. 579-598.

Vulbeau A., 2003.- Sortir de l'ombre ? Oui, mais seulement au crépuscule.... Dans Boucher M. et Vulbeau A. (Eds.) "Émergences culturelles et jeunesse populaire", Paris, L'Harmattan. 